\title{
Improving the Selection of Requirements Elicitation Techniques: A Faceted Guide
}

\author{
Rafael Crispim Ignácio ${ }^{1}$, Fabiane Barreto Vavassori Benitti ${ }^{1}$ \\ ${ }^{1}$ Departamento de Informática e Estatística - Universidade Federal de Santa \\ Catarina (UFSC) Florianópolis, Brasil
}

\begin{abstract}
In Requirements Engineering there are several techniques that can be used to elicit the requirements. In the current scenario, no sources were found in which these techniques are properly organized, facilitating their understanding in the identification of their characteristics, as well as assisting the search for techniques recommended for the context of a specific project. This paper describes the development of a organized and categorized requirements elicitation technique to support the identification of techniques appropriate to the scenario of a given project. Through a systematic mapping, 96 techniques of elicitation of requirements and 21 facets (characteristics) were identified to classify the techniques, which were organized to compose the guide presented in this paper. Through an evaluation with 30 participants, we can evidence the effectiveness of the guide to suggest techniques of elicitation, as well as its ease of use.
\end{abstract}

Resumo. Na Engenharia de Requisitos existe uma diversidade de técnicas que podem ser usadas para elicitar os requisitos. No cenário atual, não foram encontradas fontes onde essas técnicas são devidamente organizadas, facilitando o entendimento na identificação de suas características, bem como auxiliar na busca por técnicas para um determinado contexto. Este artigo descreve o desenvolvimento de um guia que organiza e categoriza as técnicas de elicitação de requisitos para suporte na identificação das técnicas apropriadas para o cenário de determinado projeto. Através de um mapeamento sistemático, 96 técnicas foram organizadas compondo o guia apresentado neste artigo. Por meio de uma avaliação com 30 participantes, nós podemos evidenciar a efetividade do guia em sugerir técnicas de elicitação, bem como o seu fácil uso.

\section{Introdução}

Uma etapa importante para a qualidade no desenvolvimento de software é a identificação e compreensão dos principais requisitos para assegurar que os produtos de software sejam totalmente compatíveis e evolutivos com o sistema [Wohlin et al. 2005]. A elicitação de requisitos é a atividade geralmente reconhecida como a mais crucial no processo de Engenharia de Requisitos [Franco and Assar 2016][Murugesan et al. 2017], sendo que sua má execução quase garantirá que o projeto final s eja u ma falha completa [Hickey and Davis 2004]. Considerando a importância da elicitação de requisitos, a melhoria em seu processo traria um impacto positivo para a indústria, como observam Hickey e Davis: "Como as falhas de projeto de software são tão desenfreadas, é bem provável que melhorar a forma como a indústria realiza a elicitação poderia ter um efeito dramático no registro de sucessos da indústria"[Hickey and Davis 2004]. 
Contudo, a elicitação de requisitos ainda permanece problemática; requisitos ausentes ou errados ainda atrasam projetos e causam custos excessivos [Franco and Assar 2016]. Deve-se considerar que para o sucesso da elicitação de requisitos é importante aplicar diferentes técnicas para projetar a solução de software, bem como é igualmente importante escolher técnicas apropriadas [Murugesan et al. 2017]. Neste contexto, uma iniciativa que possa ser utilizada tanto para auxiliar os profissionais quanto como instrumento de ensino pode contribuir para minimizar esta problemática.

Algumas iniciativas para apoiar no entendimento ou na escolha de técnicas de elicitação já foram desenvolvidas. O DTA4RE tem a proposta de ajudar a escolher as técnicas de Design Thinking mais adequadas para elicitar os requisitos, contemplando 27 técnicas [Souza et al. 2018]. O Creativity Patterns Guide é um catálogo com 60 técnicas de criatividade para apoiar a geração de ideias durante o desenvolvimento de software [Vieira et al. 2012].

Pode-se observar que as iniciativas descritas focam em uma categoria específica de técnica de elicitação, tal seja, técnicas criativas. Neste artigo é apresentado um guia, denominado RETraining, que se propõe mais amplo, sem focar em uma categoria em especial. Enquanto o DTA4RE possui uma mecanismo de pergunta e respostas (tipo wizard) para auxiliar o usuário na seleção das técnicas apropriadas, o Creativity Patterns Guide não possui nenhum mecanismo específico para esta finalidade. No presente guia é proposto o uso de facetas para apoiar a filtragem/seleção das técnicas. A Tabela 1 visa ilustrar de maneira mais detalhada as diferentes iniciativas.

Tabela 1. Comparativo entre iniciativas envolvendo organização de técnicas de elicitação de requisitos

\begin{tabular}{|c|c|c|c|}
\hline & DTA4RE & Creativity Patterns Guide & RETraining \\
\hline Contexto das técnicas & Design Thinking & Criatividade & Sem limitação \\
\hline Mecanismo para seleção & Wizard & Não contempla & Filtros baseados em facetas \\
\hline Conteúdo da técnica & $\begin{array}{l}\text { - O que é? } \\
\text { - Como aplicar? } \\
\text { - Quando usar? } \\
\text { - Exemplo } \\
\text { - Prós e contras } \\
\text { - Referências }\end{array}$ & $\begin{array}{l}\text { - Objetivo } \\
\text { - Aplicabilidade } \\
\text { - Direcionamento das ideias } \\
\text { - Participantes } \\
\text { - Informações da técnica } \\
\text { - Critérios de operação } \\
\text { - Critérios de abordagem }\end{array}$ & $\begin{array}{l}\text { - Descrição } \\
\text { - Como aplicar? } \\
\text { - Exemplo } \\
\text { - Prós e contras } \\
\text { - Referências } \\
\text { - Classificação facetada completa }\end{array}$ \\
\hline Público-alvo & Acadêmicos & Profissionais & Acadêmicos e Profissionais \\
\hline
\end{tabular}

A utilização no contexto acadêmico de um guia com técnicas de elicitação de requisitos pode evidenciar aos estudantes a existência de diversas técnicas, seus diferentes propósitos e processos, bem como apoiar a escolha de técnicas adequadas. No contexto profissional, um guia pode proporcionar aos profissionais a ampliação do seu conhecimento a partir da indicação de novas técnicas, bem como auxiliar na adequação destas ao contexto de um projeto específico. A necessidade de fornecer recursos para o aperfeiçoamento contínuo dos profissionais no que tange a Engenharia de Requisitos é evidenciada em [Malviya et al. 2017], que ao perguntar "What Questions do Requirements Engineers Ask?" observou que 55\% das questões foram sobre elicitação de requisitos, avaliação de qualidade e gerenciamento de projetos.

Neste contexto, este artigo apresenta a construção e avaliação de um guia facetado para técnicas de elicitação de requisitos. Seu principal objetivo é servir de interface para que um usuário, estudante ou profissional, possa, através dos fatores internos e externos 
do projeto, descobrir quais técnicas de elicitação de requisitos se enquadram melhor em sua situação, bem como obter uma visão geral desta técnica.

A metodologia para o desenvolvimento partiu de um mapeamento sistemático (descrito na Seção 2) para identificar as técnicas de elicitação e as facetas / características de cada técnica, cujo processo de classificação consta na Seção 3. Posteriormente, o guia foi desenvolvido (Seção 4) e, por fim, o guia foi avaliado tanto no contexto acadêmico quanto profissional utilizando abordagem GQM (Seção 5).

\section{Técnicas de elicitação de requisitos}

Embora alguns estudos [Carrizo et al. 2014] [Abbasi et al. 2015] apresentem um conjunto de técnicas de elicitação não o fazem de forma sistemática, por outro lado, estudos sistemáticos [Dieste et al. 2008] foram executados a mais de 10 anos. Desta forma, esta seção tem o objetivo de identificar as técnicas de elicitação de requisitos existentes na literatura por meio de um mapeamento sistemático, seguindo as diretrizes propostas em [Kitchenham 2010].

\subsection{Planejamento}

Duas questões foram formuladas para nortear o mapeamento sistemático:

Q1. Quais técnicas são utilizadas para elicitação de requisitos?

Q2. Quais são as características das técnicas de Elicitação de Requisitos?

Para responder estas questões foi desenvolvida a string de busca destacada abaixo com sua execução nas bases de dados IEEExplore,ACM Digital Library, Google scholar, Citeseer, ScienceDirect, Springer, Scopus e Engineering Village:

("software requirements") AND ("elicitation techniques"OR "acquisition techniques"OR "elicitation methods"OR "requirements elicitation") AND ("evaluation"OR "comparison"OR "survey"OR "comparative study"OR "review"OR "mapping"OR "categories"OR "taxonomies"OR "classifications")

Os critérios para inclusão e exclusão dos estudos foram:

- Critérios de inclusão

- Somente publicações com revisão por pares, como por exemplo, conferências e journals;

- Deve contemplar 3 ou mais técnicas de elicitação ${ }^{1}$, podendo ou não apresentar características das mesma;

- Publicado no período de 01/01/2008 até 31/12/2017 (10 anos).

- Critérios de exclusão

- Publicações que não são revisadas por pares;

- Publicações como resumos, pôsteres e tutoriais serão excluídos;

- Estudos publicados em um idioma diferente do inglês;

- Artigos que não são acessíveis publicamente ou com autorização da universidade;

- Artigo não apresenta técnicas de elicitação de requisitos de software ou apresenta menos de 3 técnicas.

\footnotetext{
${ }^{1}$ Este critério visa identificar estudos mais amplos envolvendo várias técnicas, visando obter informações comparativas para análise.
} 


\subsection{Execução}

Após a execução das buscas e coleta dos resultados foram executados dois processos, sintetizados na Figura 1.

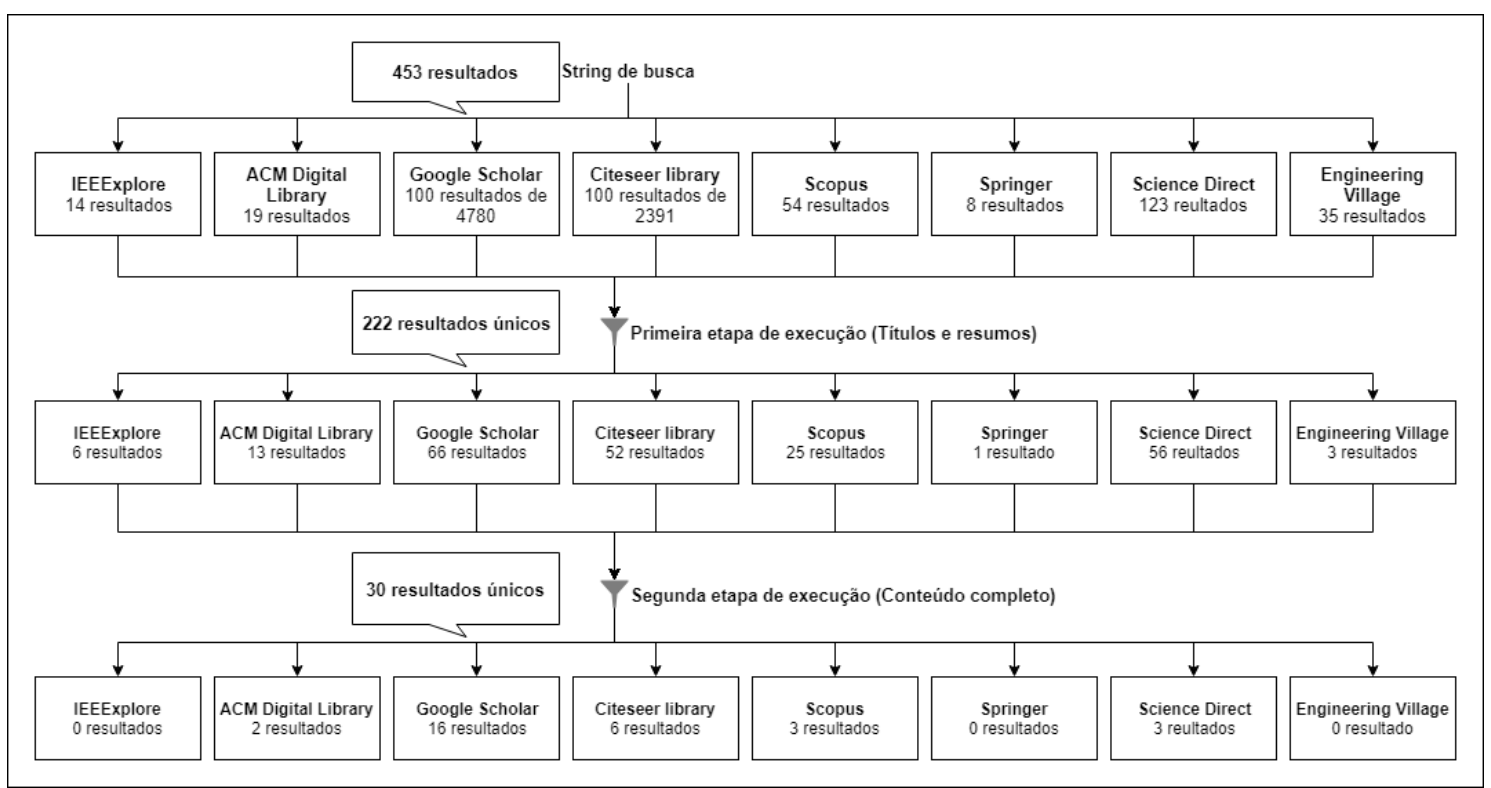

Figura 1. Detalhamento da execução da busca nas bases científicas.

1. Análise dos títulos e resumos: ao analisar os títulos e resumos, foram removidos todos artigos que não tinham relação com o tema proposto. Nesta etapa, dos 453 artigos analisados, foram selecionados 222 artigos únicos, cerca de 50\% do total.

2. Análise completa: nesta etapa foram avaliados todos os trabalhos restantes de uma maneira mais detalhada, considerando todo o conteúdo dos estudos. Dos 222 artigos que passaram pela primeira etapa, foram selecionados 30 artigos únicos, cerca de $13 \%$ do total.

\subsection{Resultados}

A execução e seleção dos artigos gerou insumos para responder as questões levantadas no planejamento do mapeamento sistemático (Seção 2.1).

\subsubsection{Q1 - Quais técnicas são utilizadas para elicitação de requisitos?}

Esta questão tem o objetivo de identificar as técnicas mais relevantes através dos trabalhos selecionados no mapeamento sistemático e obter informações inerentes às mesmas. Não existe a pretensão de atingir todas as técnicas existentes no meio acadêmico ou na indústria.

Através da análise dos estudos foram identificadas 96 técnicas de elicitação de requisitos. O número coletado demonstra que existem muitas técnicas e que a maioria é pouco explorada, $59 \%$ possui apenas 1 citação. Cerca de $21 \%$ das técnicas são encontradas em mais de 5 publicações. O estudo aponta também que a Entrevista é a técnica mais comum, encontrada em cerca de $83 \%$ das publicações selecionadas. Algumas técnicas 
podem possuir nomes diferentes, mas fazem referência ao mesmo método. Os casos identificados como similares através dos textos dos próprios autores foram unificados.

A Figura 2 apresenta, através de uma nuvem de palavras, todas as técnicas encontradas, sendo que seus respectivos tamanhos na figura representam sua frequência perante as demais. Em razão da limitação de espaço a lista completa das técnicas pode ser obtida em http://retraining.inf.ufsc.br/guia/TecnicasElicitacao. pdf.

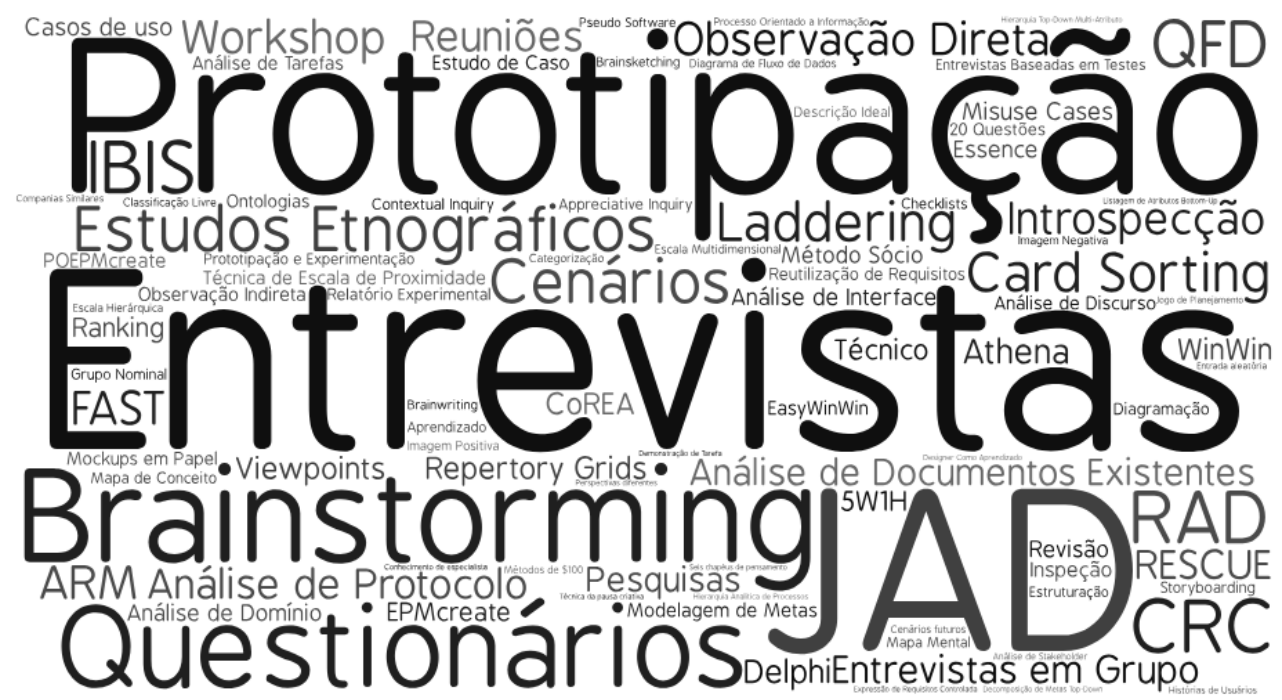

Figura 2. Nuvem de palavras das técnicas conforme a frequência

\subsubsection{Q2 - Quais são as características das técnicas de Elicitação de Requisitos?}

Esta questão tem o objetivo de identificar as características inerentes às técnicas de elicitação de requisitos a fim de agregar insumos para a criação de uma classificação facetada.

Foram identificadas 21 características através do estudo realizado. Estas informações foram extraídas de 3 das 30 publicações selecionadas. A extração das características destes 3 artigos se deve ao fato dos autores especificarem as características diretamente, relacionando com as respectivas técnicas analisadas, o que não ocorreu nos demais artigos selecionados. Estas informações foram utilizadas para a definição da classificação das técnicas catalogadas no guia (Seção 3 Tabela 2).

\section{Classificação Facetada}

Uma classificação facetada é conhecida como um esquema analítico sintético porque envolve dois processos distintos: a análise do assunto em facetas e a síntese dos elementos que constituem o mesmo, sendo, portanto, aplicável a qualquer área do conhecimento. Para definição das facetas do guia foi utilizada uma metodologia que consiste na execução de sete passos [Denton 2003]:

1. Coleta de Domínios: o domínio deste estudo foi definido através do protocolo descrito na Seção 2. 
2. Listagem de Entidades: composta pelas 96 técnicas de elicitação de requisitos disponível em http://retraining.inf.ufsc.br/guia/ Tecnicas-Elicitacao.pdf.

3. Criação de Facetas: a partir do mapeamento as características comuns às técnicas foram consideradas como possíveis facetas.

4. Arranjo de Facetas: após analisar a lista de facetas disponíveis, foram mantidas somente os casos associados diretamente às características das técnicas e não de fatores externos (Tabela 2).

5. Ordem de Citação: as facetas foram agrupadas no guia em: características gerais, elicitor, domínio do problema e stakeholder (ver Figura 3b).

6. Classificação: as facetas de cada técnica foram definidas a partir das informações disponíveis nos estudos primários selecionados.

7. Revisão, teste e manutenção: realizada a partir da avaliação (Seção 5) e continuidade do projeto.

Foram identificadas 21 facetas por meio das quais é possível classificar as técnicas de elicitação de requisitos. A Tabela 2 detalha as facetas definidas.

Tabela 2. Características relacionadas às técnicas de elicitação de requisitos

\begin{tabular}{|c|c|c|}
\hline Faceta & Descrição & Valores \\
\hline Tipo de técnica [Abbasi et al. 2015] & Identifica qual a forma de extração de requisitos & Direta ou Indireta \\
\hline Tipo de dado [Abbasi et al. 2015] & Identifica o tipo do dado extraído & Qualitativo ou Quantitativo \\
\hline Comunicação [Abbasi et al. 2015] & Identifica a direção da comunicação entre os envolvidos & Unidirecional ou Bidirecional \\
\hline $\begin{array}{ll}\text { Compreensão } & \text { do domínio } \\
\text { [Abbasi et al. 2015] } & \end{array}$ & Identifica a necessidade de entendimento do domínio da aplicação & Sim ou Não \\
\hline Fonte principal [Al Mrayat et al. 2013] & Fonte principal responsável por fornecer insumos ou conduzir a técnica & $\begin{array}{l}\text { Analista com conhecimento no domínio, Facili- } \\
\text { tador externo, Observador, Documentação, Es- } \\
\text { pecialista ou Analistas e Stakeholders }\end{array}$ \\
\hline Treinamento [Carrizo et al. 2014] & Treinamento prévio e prática do elicitor com a técnica de elicitação & Alto, Baixo ou Nenhum \\
\hline $\begin{array}{l}\text { Experiência } \\
\text { elicitor[Carrizo et al. 2014] do }\end{array}$ & Número de projetos que o elicitor realizou atividades de elicitação & Alto, Médio ou Baixo \\
\hline $\begin{array}{l}\text { Experiência } \\
\text { técnicas[Carrizo et al. 2014] }\end{array}$ & $\begin{array}{l}\text { Número de projetos que o elicitor realizou atividades com determinada } \\
\text { técnica }\end{array}$ & Alto, Baixo ou Nenhum \\
\hline $\begin{array}{lr}\text { Familiaridade rom } & \text { com } \\
\text { domínio[Carrizo et al. 2014] }\end{array}$ & $\begin{array}{l}\text { Número de projetos no mesmo domínio ou no domínio de conheci- } \\
\text { mento do elicitor }\end{array}$ & Alto, Baixo ou Nenhum \\
\hline Pessoas por sessão[Carrizo et al. 2014] & Número de indivíduos que podem participar da sessão de elicitação & Individual, Grupo ou Em massa \\
\hline $\begin{array}{ll}\text { Consenso } & \text { entre } \\
\text { stakeholders[Carrizo et al. 2014] }\end{array}$ & Acordo inicial entre os envolvidos & Alto ou Baixo \\
\hline $\begin{array}{l}\text { Interesse } \\
\text { stakeholder[Carrizo et al. 2014] }\end{array}$ & Interesse dos envolvidos em participar das sessões de elicitação & Alto, Baixo ou Nenhum \\
\hline Especialidade[Carrizo et al. 2014] & Experiência do informante no problema ou no domínio & Especialista, Bem informado ou Iniciante \\
\hline Articulação[Carrizo et al. 2014] & Habilidade do informante em passar seu conhecimento & Alto, Médio ou Baixo \\
\hline $\begin{array}{l}\text { Disponibilidade } \\
\text { tempo[Carrizo et al. 2014] }\end{array}$ & Tempo disponível do informante & Alto ou Baixo \\
\hline Local/Acessibilidade[Carrizo et al. 2014] & Localização do informante em relação ao elicitor & Longe ou Perto \\
\hline $\begin{array}{l}\text { Tipo de informação } \\
\text { elicitar[Carrizo et al. 2014] }\end{array}$ & Tipo de informação que a técnica pode levantar & Estratégica, Tática ou Básica \\
\hline $\begin{array}{l}\text { Nível de informação dis- } \\
\text { ponível[Carrizo et al. 2014] }\end{array}$ & Informação disponível antes da execução da técnica & Superior, Inferior ou Nenhum \\
\hline $\begin{array}{l}\text { Definição } \\
\text { problema[Carrizo et al. 2014] }\end{array}$ & Clareza dos objetivos e escopo do projeto & Alto ou Baixo \\
\hline $\begin{array}{l}\text { Restrição } \quad \text { de } \quad \text { tempo } \\
\text { projeto[Carrizo et al. 2014] }\end{array}$ & Tempo disponível para aplicação da técnica no projeto & Alto, Médio ou Baixo \\
\hline Tempo de processo[Carrizo et al. 2014] & Etapa de pré-sessão do processo de elicitação & Início, Meio ou Fim \\
\hline
\end{tabular}

\section{Apresentação do Guia}

O guia objetiva que tanto estudantes da área de tecnologia da informação quando profissionais atuantes na área possam encontrar de forma estruturada informações sobre técnicas de elicitação de requisitos. Tendo em vista que o tempo em sala de aula destinado a este tema ou o tempo destinado à aprendizagem em uma empresa de desenvolvimento de software são relativamente curtos, a existência de um guia facetado que torne as informações acessíveis tende a facilitar o aprendizado de novas técnicas.

Neste primeiro momento foram priorizadas a publicação de 12 técnicas de elicitação, selecionadas de acordo com o percentual de facetas que se dispunha 
informações a respeito das técnicas. Desta forma, as técnicas atualmente disponíveis são: entrevista, prototipação, brainstorming, card sorting, entrevista em grupo, JAD, laddering, observação direta, análise de protocolo, questionários, repertory grids e cenários (Figura 3a). Para filtrar as técnicas a partir das 21 facetas identificadas, visando obter técnicas recomendadas de acordo com características específicas de um projeto, o guia dispõe de um painel de filtragem (Figura 3b).

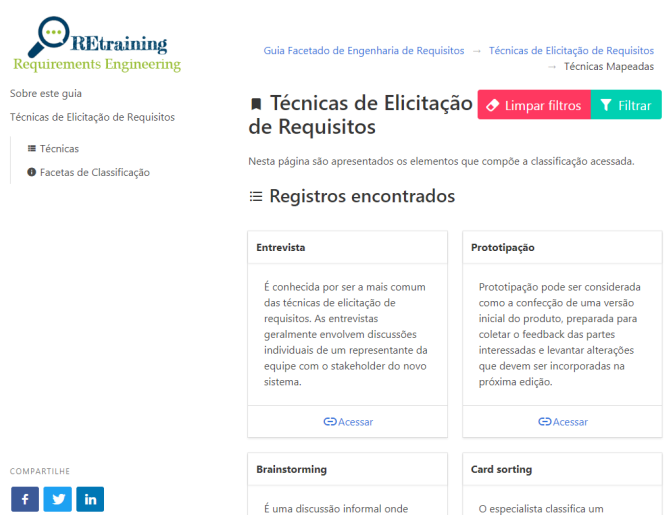

(a) Listagem das técnicas

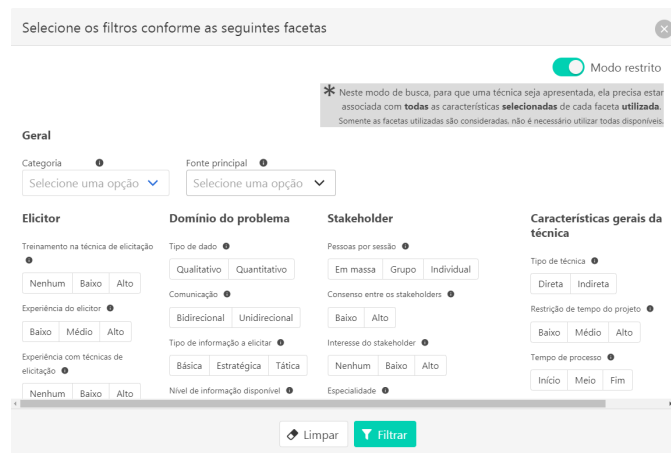

(b) Painel para filtragem

Figura 3. Telas do guia RETraining

\section{Avaliação}

A avaliação do guia foi realizada utilizando a abordagem GQM (Goal-QuestionMetric) [Caldiera and Rombach 1994], observando as etapas de planejamento, execução e interpretação dos resultados.

\subsection{Planejamento}

O primeiro objetivo a ser avaliado diz respeito a qualidade e organização do conteúdo (formulado conforme proposto em [Caldiera and Rombach 1994]):

Objetivo de medição 1: Analisar o guia de técnicas de elicitação de requisitos com o propósito de avaliar a facilidade de entendimento com respeito a apresentação de novas técnicas e compreensão das mesmas do ponto de vista de profissionais e acadêmicos no contexto da elicitação de requisitos.

O segundo objetivo diz respeito a usabilidade da ferramenta(formato proposto por [Caldiera and Rombach 1994]):

Objetivo de medição 2: Analisar o guia de técnicas de elicitação de requisitos, com o propósito de avaliar a facilidade de utilização da ferramenta, com respeito a navegação e disposição das informações, do ponto de vista de usuário, no contexto da aplicação. Para responder este objetivo foi utilizada a escala SUS que resulta em um indicador que corresponde ao nível de usabilidade da ferramenta avaliada [McLellan et al. 2012].

Para avaliar os objetivos propostos foram definidas as questões e métricas detalhadas na Tabela 3. 
Tabela 3. Questões e métricas para os objetivos

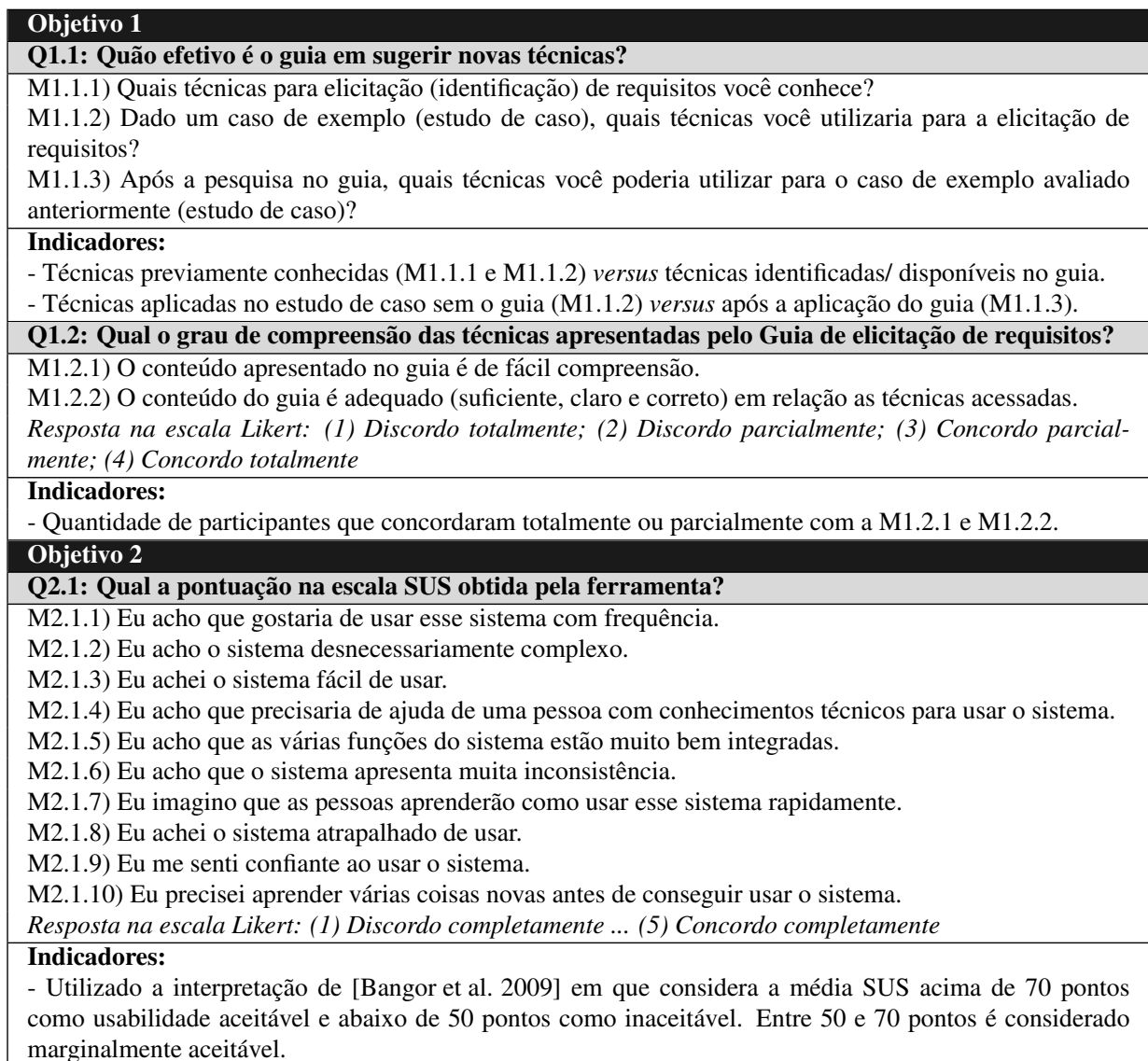

A partir da definição destes objetivos e métricas foi elaborado um questionário ${ }^{2}$ para coleta dos dados. O questionário é composto por 24 perguntas divididas em 5 seções:

1. Identificação do participante: solicita alguns dados para definir o perfil do participante, como por exemplo, formação, perfil profissional e experiência com engenharia de requisitos;

2. Conhecimento prévio: solicita ao participante informar as técnicas de elicitação que ele conhece e apresenta um estudo de caso para que o participante identifique técnicas que ele utilizaria no contexto específico;

3. Guia: apresenta o link do guia e solicita ao participante explorar o conteúdo;

4. Conhecimento posterior: solicita ao participante informar as técnicas que ele utilizaria para o estudo de caso (mesmo da seção 2) com auxílio do guia, bem como solicita avaliar a compreensão e adequação do conteúdo apresentado no guia;

5. Usabilidade: solicita ao participante preencher as questões do questionário SUS (Tabela 3).

\subsection{Execução}

A coleta de dados foi feita através de entrevistas estruturadas, a qual desenvolve-se a partir de uma relação fixa de perguntas (questionário descrito na Seção 5.1), cuja ordem e redação permanece invariável para todos os participantes, permitindo a análise estatística

\footnotetext{
${ }^{2}$ Disponível em https://forms.gle/VWfGNrC7Dje1oxHt5
} 
dos dados [Gil 2008]. A amostragem dos participantes foi realizada por conveniência observando uma estratificação pelo perfil, buscando equiparar a quantidade de participantes classificados como profissionais e acadêmicos, sendo que os critérios adotados para classificar os participantes foram:

- Perfil Acadêmico: curso concluído ou em andamento na área de TI; ter cursado ao menos uma disciplina que aborde o tema de engenharia de requisitos; e ter menos de 1 ano de experiência com a atividade de levantamento/análise de requisitos.

- Perfil Profissional: ter 1 ano ou mais de experiência com a atividade de levantamento/análise de requisitos.

Ao todo foram entrevistadas 30 pessoas, sendo $16 \mathrm{com}$ perfil acadêmico e $14 \mathrm{com}$ perfil profissional. Dos profissionais, 5 com experiência entre 1 e 3 anos (considerados experientes) e 9 com mais de 3 anos de experiência em levantamento e análise de requisitos (considerados expert).

\subsection{Resultados}

Esta seção apresenta os resultados obtidos para cada questão planejada na avaliação.

\subsubsection{Q1.1. Quão efetivo é o guia em sugerir novas técnicas?}

Para responder sobre a efetividade do guia em sugerir novas técnicas foi perguntado aos participantes acerca do tema, antes e depois da apresentação do guia. Inicialmente, foram questionadas quais técnicas eram conhecidas pelo entrevistado, na forma de questão aberta. Em um segundo momento, a partir da contextualização de um estudo de caso, foi questionado aos participantes quais técnicas consideravam adequadas para a elicitação de requisitos do cenário exposto. A Figura 4 apresenta (i) as técnicas citadas por cada perfil, (ii) a frequência de citações e, (iii) em laranja as técnicas já disponíveis no guia, em vermelho as identificadas a partir do mapeamento sistemático e ainda não incluídas no guia e, em cinza, as técnicas não identificadas no mapeamento (neste grupo são contemplados todos os termos citados na avaliação, sem julgamento da sua adequação como técnica de elicitação).

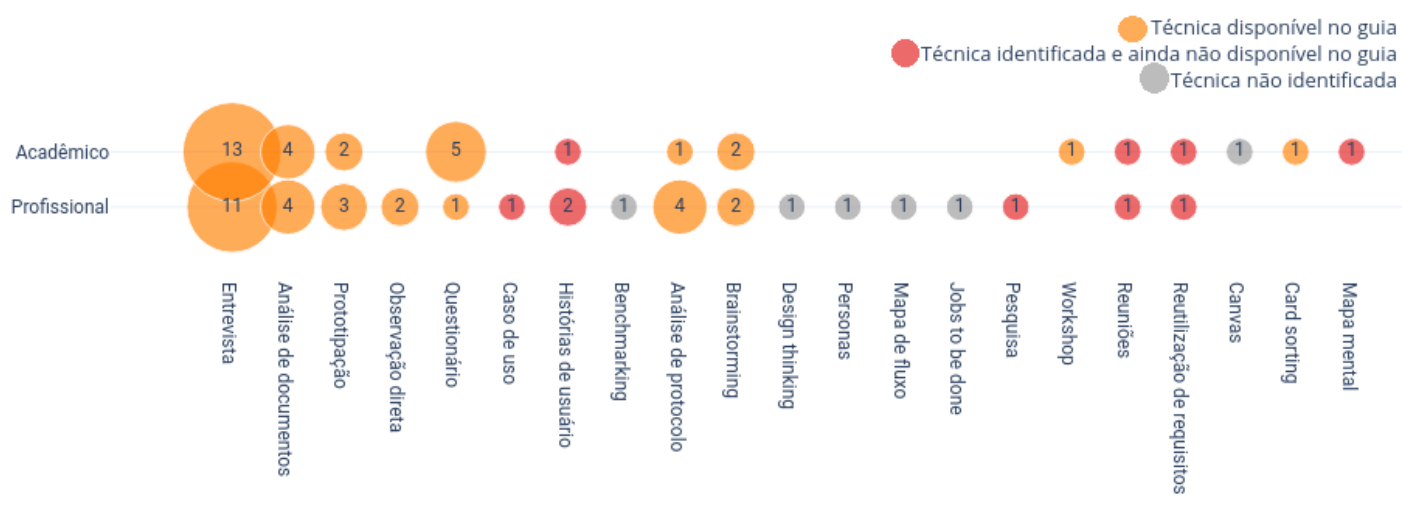

Figura 4. Técnicas citadas antes da apresentação do guia

Os 30 participantes da avaliação citaram ao todo 21 diferentes técnicas, das quais 9 já estão disponíveis no guia; 6 foram identificadas no mapeamento sistemático e ainda 
não constam do guia e 6 técnicas não foram identificadas no mapeamento (Figura 4). A técnica de entrevista foi a mais lembrada entre os participantes, seguida por análise de documentos.

Após os participantes explorarem o guia, o estudo de caso foi reapresentado e novamente foi questionado quais técnicas poderiam ser utilizadas. A Figura 5 ilustra a quantidade de respostas adequadas antes e após a utilização do guia.

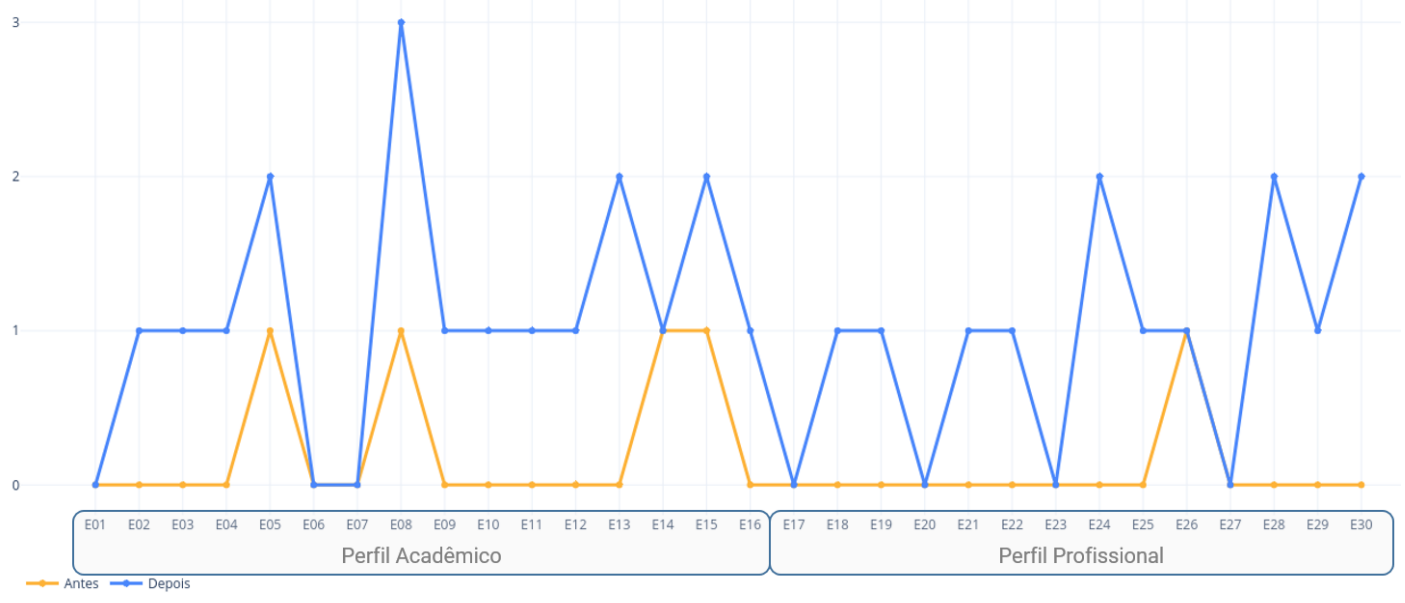

Figura 5. Quantidade de técnicas consideradas adequadamente identificadas para o estudo de caso antes e depois do uso do guia

A interpretação das características presentes no estudo de caso e a adequação das técnicas era parte do processo avaliativo. Na maioria dos casos, as respostas não foram adequadas ao problema apresentado antes do acesso ao guia. Apenas 5 participantes citaram uma técnica esperada como uma opção válida (Figura 5). Contudo, observou-se que $70 \%$ dos participantes reviram sua resposta após a utilização do guia, indicando uma certa influência do guia sobre suas respostas. Neste contexto, formulou-se as seguintes hipóteses:

H0: O guia proposto não auxilia o usuário a identificar técnicas adequadas para um projeto.

H1:O guia proposto auxilia o usuário a identificar técnicas adequadas para um projeto.

O teste de Wilcoxon (Wilcoxon signed-ranks test) foi utilizado por ser um método não-paramétrico para comparação de duas amostras pareadas. O teste apontou pi..05, permitindo rejeitar $\mathrm{H} 0$ e aceitar $\mathrm{H} 1$, concluindo que, para a amostra considerada, o guia proposto auxilia o usuário a identificar técnicas adequadas para um projeto. Buscou-se também aplicar o teste de Wilcoxon considerando apenas a amostra de acadêmicos e profissionais separadamente, visando identificar se o guia auxiliou tanto os usuários com um perfil quanto outro. Os resultados do teste estatístico permitem identificar que o guia auxiliou tanto acadêmicos quanto profissionais ( $\mathrm{p}_{i} .05$ para ambas amostras). 


\subsubsection{Q1.2. Qual o grau de compreensão das técnicas apresentadas pelo Guia de elicitação de requisitos?}

Complementando a avaliação do conteúdo apresentado, foram efetuadas perguntas acerca da qualidade e adequação do conteúdo produzido e apresentado no guia (Tabela 3). Para a primeira métrica desta questão, obteve-se como resultado que $63 \%$ dos participantes concordaram totalmente que a ferramenta possui um conteúdo de fácil compreensão, os demais entrevistados concordaram parcialmente com essa afirmação. A proporção de entrevistados que concordaram totalmente com a afirmação de que o conteúdo é adequado para as técnicas acessadas foi de $73 \%$, sendo que $20 \%$ concordaram parcialmente e outros $7 \%$ discordaram parcialmente. A Figura 6 ilustra o resultado.

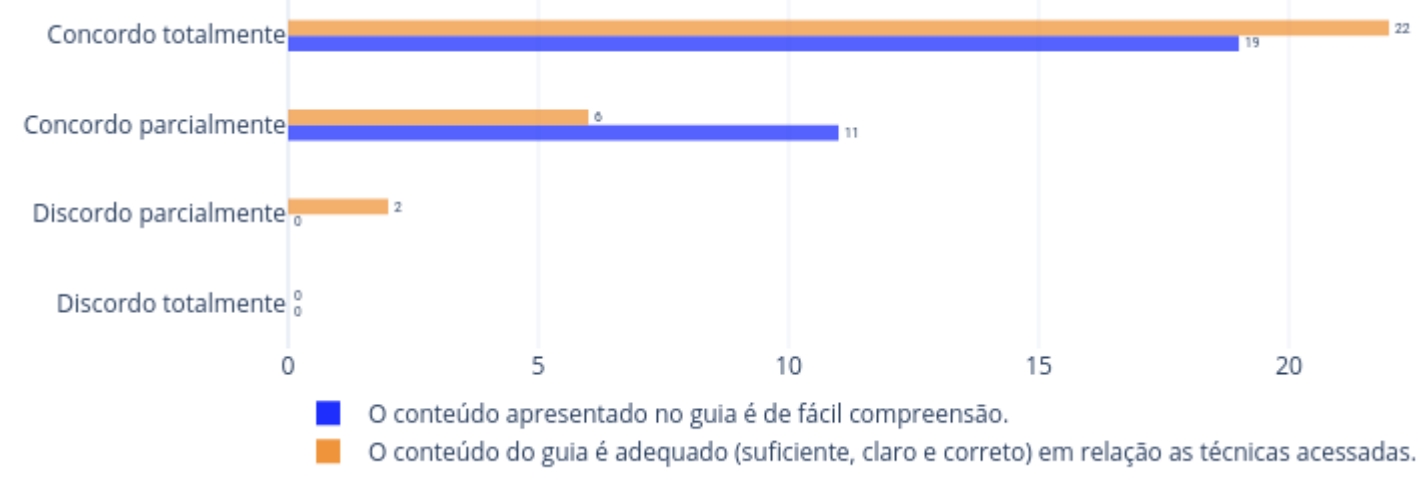

Figura 6. Avaliação quanto a compreensão das técnicas apresentadas pelo guia

\subsubsection{Q2.1. Qual a pontuação na escala SUS obtida pela ferramenta?}

Quanto ao segundo objetivo de avaliação (Tabela 3) foram analisados os aspectos de usabilidade associados à ferramenta desenvolvida. Para responder a pergunta relacionada a usabilidade, optou-se em utilizar a escala SUS, conforme apresentado na Tabela 3. A média da avaliação SUS do guia resultou em 86 pontos. Este valor indica que o guia foi avaliado com um nível de usabilidade aceitável pelos participantes da avaliação [Bangor et al. 2009]. A Figura 7 apresenta o indicador SUS obtido a partir da avaliação de cada participante.

\subsection{Ameaças à validade}

No que tange as ameaças à validade do mapeamento sistemático (Seção 2) considerase que algum estudo relevante pode não ter sido identificado, contudo minimizou-se esta ameaça contemplando sinônimos e termos relacionados na string, bem como contemplado 8 bases de dados. Outra ameaça se refere ao fato da etapa de execução ter sido realizada principalmente pelo primeiro autor deste artigo, de forma que uma interpretação equivocada dos critérios sobre um estudo pode gerar uma alteração nos resultados apresentados, esta ameaça foi minimizada com os casos de incerteza sendo avaliados também pelo segundo autor deste artigo.

No que tange a avaliação (seção 5) pode-se citar as seguintes ameaças: (i) a amostra de 30 participantes, pois tanto o número é pequeno quanto a amostragem por 


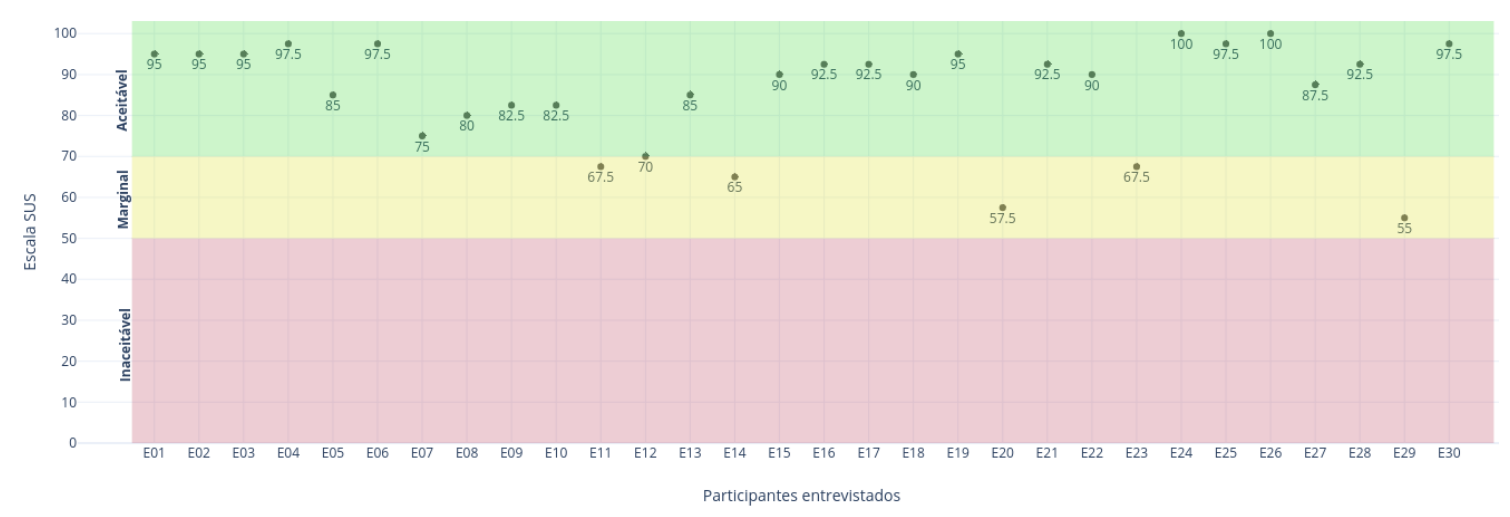

Figura 7. Avaliação da usabilidade do guia

conveniência, adotada nesta pesquisa, constitui o menos rigoroso de todos os tipos de amostragem [Gil 2008]; (ii) se por um lado a entrevista oferece flexibilidade, posto que o entrevistador pode elucidar dúvidas, por outro, tem-se a ameaça da presença do pesquisador influenciar o entrevistado. Minimizou-se esta ameaça com o preenchimento das questões, após o uso do guia, pelo próprio participante, sem interferência do entrevistador; e (iii) a identificação das técnicas conhecidas pelos participantes foi realizada através de questão aberta, requerendo, portanto, uma consolidação das técnicas citadas em relação a sua nomenclatura, podendo ocorrer um mapeamento indevido nesta etapa. Contudo, como a coleta foi realizada através de entrevista, este método permitiu ao entrevistador se apropriar do teor da técnica relatada, facilitando a tarefa de consolidação e minimizando esta ameaça.

\section{Conclusões}

Esta pesquisa colaborou com evidências de que existem diversas técnicas para elicitação de requisitos, tendo identificado 96 técnicas conforme os critérios aplicados no mapeamento sistemático. Foram encontradas desde técnicas bem conhecidas até técnicas que estão em fase de experimentação. Estes dados corroboram com as citações de outros autores mencionadas no início deste artigo (Seção 1) referente a existência de um número considerável de técnicas e, portanto, evidencia a necessidade de um guia sintetizando tais informações.

O mapeamento sistemático também se mostrou útil para apoiar uma classificação facetada, a qual se demonstrou aplicável para o problema em questão. A seleção das características a serem tratadas como facetas compõem, por si só, uma contribuição do presente estudo. Este estudo identificou e utilizou 21 características para filtrar e recomendar técnicas de elicitação de requisitos.

Até o presente momento, o guia classifica 12 técnicas de elicitação de requisitos, um número relativamente baixo se comparado às 96 técnicas identificadas, contudo, demonstrou ser uma quantidade aceitável para viabilizar uma avaliação inicial e direcionar esforços futuros.

A avaliação do guia apresentou resultados positivos tanto para os conteúdos produzidos quanto para a aplicação desenvolvida, demonstrando que as decisões tomadas foram assertivas no decorrer da execução. A avaliação da efetividade do guia permitiu 
(i) identificar 6 técnicas não resultantes do mapeamento sistemático, elevando para 102 as técnicas identificadas nesta pesquisa, considerando que as técnicas informadas pelos participantes de fato se referem a elicitação de requisitos; e (ii) constatar, mesmo que de maneira preliminar, a efetividade do guia em sugerir novas técnicas. Também observase que o guia com seu painel para filtragem por facetas foi considerado fácil de utilizar, obtendo avaliação de 86 pontos na escala SUS.

Os resultados obtidos até o momento encorajam a evolução desta pesquisa, tendose como trabalhos futuros a inclusão de novas técnicas de elicitação no guia, um mecanismo de recomendação de técnicas complementares a partir do interesse por uma técnica específica, bem como a extensão do guia para contemplar também outras etapas do processo, como por exemplo, técnicas para especificação de requisitos.

\section{Referências}

Abbasi, M. A., Jabeen, J., Hafeez, Y., Batool, D., and Fareen, N. (2015). Assessment of requirement elicitation tools and techniques by various parameters. Software Engineering, 3(2):7-11.

Al Mrayat, O. I., Norwawi, N., and Basir, N. (2013). Requirements elicitation techniques: comparative study. International Journal of Recent Development in Engineering and Technology, 1(3):1-10.

Bangor, A., Kortum, P., and Miller, J. (2009). Determining what individual sus scores mean: Adding an adjective rating scale. Journal of usability studies, 4(3):114-123.

Caldiera, V. R. B.-G. and Rombach, H. D. (1994). Goal question metric paradigm. Encyclopedia of software engineering, 1:528-532.

Carrizo, D., Dieste, O., and Juristo, N. (2014). Systematizing requirements elicitation technique selection. Information and Software Technology, 56(6):644-669.

Denton, W. (2003). How to make a faceted classification and put it on the web. See http://www.miskatonic.org/library/facet-web-howto.html.

Dieste, O., Juristo, N., and Shull, F. (2008). Understanding the customer: what do we know about requirements elicitation? IEEE Software, 25(2):11-13.

Franco, A. and Assar, S. (2016). Leveraging creativity techniques in requirements elicitation: A literature review. Requirements Engineering Magazine, (2016-2).

Gil, A. C. (2008). Métodos e técnicas de pesquisa social. 6. ed. Editora Atlas SA.

Hickey, A. M. and Davis, A. M. (2004). A unified model of requirements elicitation. Journal of Management Information Systems, 20(4):65-84.

Kitchenham, B. (2010). What's up with software metrics?-a preliminary mapping study. Journal of systems and software, 83(1):37-51.

Malviya, S., Vierhauser, M., Cleland-Huang, J., and Ghaisas, S. (2017). What questions do requirements engineers ask? In 2017 IEEE 25th International Requirements Engineering Conference (RE), pages 100-109.

McLellan, S., Muddimer, A., and Peres, S. C. (2012). The effect of experience on system usability scale ratings. Journal of usability studies, 7(2):56-67. 
Murugesan, L. K., Hoda, R., and Salcic, Z. (2017). Identifying design features using combination of requirements elicitation techniques. In Design and Innovation in Software Engineering (DISE), 2017 IEEE/ACM 1st Int. Workshop on, pages 6-12. IEEE.

Souza, A. F., Ferreira, B., Correa, L., Marczak, S., and Conte, T. (2018). Design thinking assistant for requirements elicitation.

Vieira, E. R., Alves, C., and Duboc, L. (2012). Creativity patterns guide: Support for the application of creativity techniques in requirements engineering. In Winckler, M., Forbrig, P., and Bernhaupt, R., editors, Human-Centered Software Engineering, pages 283-290, Berlin, Heidelberg. Springer Berlin Heidelberg.

Wohlin, C. et al. (2005). Engineering and managing software requirements. Springer Science \& Business Media. 